\title{
Transient receptor potential ankyrin 1 (trpa1) mediates il-1 $\beta$-induced apoptosis in rat chondrocytes via calcium overload and mitochondrial dysfunction
}

\author{
Songjiang Yin, Li Zhang, Liang Ding, Zhengquan Huang, Bo Xu, XiaoChen Li, Peimin Wang and Jun Mao*
}

\begin{abstract}
Background: Chondrocyte apoptosis is a central feature in the progression of osteoarthritis (OA), and would be triggered by sustained elevation of intracellular calcium ion $\left(\mathrm{Ca}^{2+}\right)$, also known as a cellular second messenger. Transient receptor potential ankyrin 1 (TRPA1) is a membrane-associated cation channel, and the activation of which causes an influx of cation ions, in particularly $\mathrm{Ca}^{2+}$, into the activated cells. Therefore, we investigate the potential role of TRPA1 in mediating $\mathrm{Ca}^{2+}$ influx to promote chondrocyte apoptosis in OA.
\end{abstract}

Methods: The expression of TRPA1 in interleukin (IL)-1 $\beta$-treated rat chondrocytes was assessed by Polymerase chain reaction (PCR) and Western blot (WB), and the functionality of TRPA1 channel by $\mathrm{Ca}^{2+}$ influx measurements.

Meanwhile, the chondrocyte apoptosis in IL-1 $\beta$-treated cells was measured by TUNEL assay and flow cytometry. The measurement of mitochondrial membrane potential and apoptosis-associated proteins after inhibition of TRPA1 were also performed in IL-1 $\beta$-treated rat chondrocytes.

Results: After being induced by IL-1 $\beta$, the gene and protein expression of TRPA1 was increased in the dosedependent manner. Meanwhile, $\mathrm{Ca}^{2+}$ influx mediated by TRPA1 in rat chondrocytes was also enhanced.

Pharmacological inhibition of TRPA1 downregulated the apoptotic rate in IL-1 $\beta$-treated rat chondrocytes. In addition, the membrane potential depolarization was improved and significantly increased expression of apoptosisassociated proteins also reduced by the TRPA1 antagonist.

Conclusions: We found the IL-1 $\beta$ caused the increased functional expression of TRPA1, the activation of which involved IL-1 $\beta$-induced apoptosis in rat chondrocytes. The potential mechanism may be linked to the intracellular calcium overload mediated by TRPA1 and attendant mitochondrial dysfunction.

Keywords: Chondrocyte apoptosis, Osteoarthritis, Transient receptor potential ankyrin 1, Calcium overload, Mitochondrial dysfunction

\section{Introduction}

Knee Osteoarthritis (OA), the most common form of arthritis, is a major cause of joint pain, activity limitation, and physical disability in the elderly [1]. Pathologically, the disease is characterized by the progressive degeneration of articular cartilage [2]. Chondrocytes, the resident cells in articular cartilage, play an important role in the homeostasis of cartilage metabolism [3], and the compromising of

\footnotetext{
* Correspondence: junmao1978@hotmail.com

Departments of orthopedics, Affiliated Hospital of Nanjing University of TCM, Road Hanzhong 155\#, Nanjing, Jiangsu Province, China
}

chondrocyte function and survival would lead to the failure of articular cartilage [4]. In OA joint, the cartilage becomes hypocellular and lacunar emptying, which suggest the chondrocyte apoptosis is a central feature in the disease progression [5]. Therefore, the modification of chondrocyte apoptosis has gradually been considered as one of the potential therapeutic targets for OA [6].

Calcium ion $\left(\mathrm{Ca}^{2+}\right)$ is a major intracellular second messenger considered as a key regulator of cell survival [7]. It is well known that sustained elevation of intracellular $\mathrm{Ca}^{2+}$ was capable of inducing $\mathrm{Ca}^{2+}$ entry-dependent ROS production [8],

(c) The Author(s). 2018 Open Access This article is distributed under the terms of the Creative Commons Attribution 4.0 International License (http://creativecommons.org/licenses/by/4.0/), which permits unrestricted use, distribution, and 
mitochondrial depolarization [9] and then apoptosis [10]. Transient receptor potential (TRP) ion channels are a large family of membrane-associated cation channels, which most permeable to $\mathrm{Ca}^{2+}$ [11]. Therefore, the activation of TRP channels serves as an important $\mathrm{Ca}^{2+}$ entry pathway contributing to fluctuation in intracellular $\mathrm{Ca}^{2+}$ and subsequent signal pathways [12]. Recently, emerging evidences also suggested the vital role of overload $\mathrm{Ca}^{2+}$ influx through TRP ion channels in cell deaths [13], such as hypoxia-induced apoptosis in H9C2 Cells [14], acidic solution-triggered apoptosis in synovial fibroblasts [15], and even monoiodoacetic acid (MIA) -mediated apoptosis in chondrocytes [16].

Transient receptor potential ankyrin 1 (TRPA1), a member of TRP ion channel, mainly mediates pain and inflammation [17], widely expressed in neuronal and non-neuronal cells [18]. The activation of TRPA1 causes an influx of $\mathrm{Ca}^{2+}$ into the activated cells, which has been shown to involve in biological processes as diverse as factors secretion [19], gene transcription [20] and cell death [21]. Recently, the effect of TRPA1 mediating synovial inflammation and cartilage degradation has been well recognized [22, 23], which was attributed mainly to a TRPA1-induced imbalance between the production of catabolic, anabolic, and inflammatory mediators [19, 24]. However, to date, no attention had been paid to define the relationship between TRPA1 and chondrocyte apoptosis. Therefore, we aimed to investigate whether or not the potential regulatory effect of TRPA1 in mediating IL-1 $\beta$ - induced apoptosis in rat chondrocytes.

\section{Methods and materials}

\section{Isolation of rat chondrocytes}

Rat chondrocytes were isolated as described [25]. In brief, the pieces of articular cartilage were aseptically dissected and separated from the tibial plateau. Then the cartilage was washed with phosphate-buffered saline (PBS) and cut into small pieces, which were digested in $0.2 \%$ collagenase type II for $6 \mathrm{~h}$. After terminating digestion and filtrating, this suspension was then centrifuged at $1500 \mathrm{rpm}$ for 6 min to collect the chondrocytes which cultured in a 10 $\mathrm{cm}^{2}$ culture flask (Corning, NY, USA) in DMEM supplemented with $10 \%$ fetal bovine serum (Gibco, MA, USA), $1 \%$ penicillin/ streptomycin (Invitrogen, CA, USA) at $37^{\circ}$ $\mathrm{C}$ in $5 \% \mathrm{CO}^{2}$. All the experiments were conducted using rat chondrocytes from passages $2-3$.

\section{In vitro treatment of rat chondrocytes}

Rat chondrocytes were seeded in $2 \mathrm{~mL}$ of medium in a 6 -well plate. Each culture was treated with IL-1 $\beta$ (PEPROTECH, USA) in the dose of $0 \mathrm{ng} / \mathrm{ml}, 0.1 \mathrm{ng} / \mathrm{ml}, 1 \mathrm{ng} / \mathrm{ml}, 5$ $\mathrm{ng} / \mathrm{ml}, 10 \mathrm{ng} / \mathrm{ml}$ for $12 \mathrm{~h}$ and $24 \mathrm{~h}$ respectively and incubated at $37^{\circ} \mathrm{C}$ in $5 \% \mathrm{CO}^{2}$. In addition, chondrocytes were also treated with IL-1 $\beta$ following pre-treated with the specific TRPA1 antagonist HC-030031 (Sigma, USA) at the dose of $100 \mu \mathrm{M}$ which has been established previously [24], in order to detect apoptosis-associated proteins, mitochondrial membrane potential and apoptosis.

\section{Real-time PCR}

RNA was isolated from treated chondrocytes with Trizol. RNA concentration and purity were measured by spectrophotometer, A260nm/A280nm ratio being 1.8 2.0. cDNA was synthesized from $2 \mu \mathrm{g}$ of total RNA using random primers and M-MLV Reverse Transcriptase (Invitrogen, Carlsbad, CA, USA). RNA expression was measured with a SYBR Green PCR kit using ABI 7500 Real-Time PCR system (Applied Biosystems, CA, USA) according to the manufacturer's instructions. The relative expression level of the target gene was calculated using the $2^{-\Delta \Delta \mathrm{CT}}$ method.

\section{Protein extraction and western blotting}

Western blotting was performed as previously described [20]. Blots were incubated with primary antibodies including TRPA1 (Novus Biologicals, USA, dilution $2 \mu \mathrm{g} / \mathrm{ml}$ ), Bax (Proteintech, USA, dilution 1:1000), cytochrome c (Proteintech, USA, dilution 1:500), PARP1 (Proteintech, USA, dilution 1:500).

\section{$\mathrm{Ca}^{2+}$-influx measurements}

Rat chondrocytes were loaded with $5 \mu \mathrm{M}$ fluo-3-acetoxy methyl ester (Fluo-3-AM, Sigma, USA) and Pluronic F-127 (KeyGEN BioTECH, China) containing $25 \mathrm{mM}$ HEPES (Sigma, USA) for $30 \mathrm{~min}$ at $37^{\circ} \mathrm{C}$ in the dark, then washed twice with $\mathrm{PBS}$ to remove extracellular Fluo-3-AM. Imaging was performed using Leica inverted microscope and analyzed with ImageJ software. The measured average fluorescence intensity of each cell in the field (F) was normalized with the non-specific background fluorescence (F0) to obtain the fluorescence intensity (F/F0).

\section{Caspase- 3 and Caspase- 9 activity detection}

The caspase- 3 and caspase- 9 activity was detected with Caspase-3 and -9 Activity Assay Kit (Solarbio, Beijing). The specific steps were conducted according to the manufacturer's instructions. In brief, $1 \times 10^{6}$ cells were lysed in $50 \mu \mathrm{l}$ lysis buffer on ice for $10 \mathrm{mins}$, and centrifuged with $12,000 \mathrm{~g}$ at $4{ }^{\circ} \mathrm{C}$ for $10 \mathrm{~min}$ to collect the supernatant. Protein concentrations were detected using the Bradford method, ensuring that the protein concentration was $1-3 \mu \mathrm{g} / \mu \mathrm{l}$. The optical density of specimen was read on a microplate reader (PerkinElmer, USA) at $405 \mathrm{~nm}$. The percentage of caspase- 3 and caspase- 9 activity changes was calculated by the radio of OD405 of the experimental wells to that of the normal wells. 


\section{Measurement of mitochondrial membrane potential}

Changes in mitochondrial membrane potential were assessed by mitochondrial membrane potential assay kit with JC-1 (Beyotime, China). Briefly, cells were seeded at a density of $1 \times 10^{6}$ cells per well onto 6-well plates and incubated with the medium containing JC-1 working liquid at $37{ }^{\circ} \mathrm{C}$ for 20 min. The cells treated with carbonylcyanidem-chlorophenylhydrazone (CCCP, 10uM, Beyotime, China) were used as positive control and the fluorescence images were observed using a fluorescent microscope (Leica, Germany). In addition, the cells were also collected and washed twice with PBS, and then analyzed by flow cytometry (Becton Dickinson, USA).

\section{TUNEL}

TUNEL assay was performed on the IL-1 $\beta$-treated cells using the one-step TUNEL apoptosis assay kit (Beyotime, Shanghai, China) according to the manufacturer's instructions. Briefly, the cells were treated by TUNEL for $1 \mathrm{~h}$ at $37 \mathrm{C}$. The cells were imaged under a fluorescent microscope (Leica, Germany) by using $488 \mathrm{~nm}$ excitation and $530 \mathrm{~nm}$ emission. The cells with green fluorescence were defined as apoptotic cells.

\section{Annexin V-FITC/PI double staining assay}

After treatment, chondrocytes were stained with Annexin-V and propidium iodide (PI) using the Annexin-V-FITC kit (FMSAV-100, FcMACS, Nanjing, China) according to the protocol of the company. In brief, the chondrocytes were suspended in binding buffer with $1 \times 10^{6}$ cells $/ \mathrm{ml}$. Afterward, $5 \mu \mathrm{l}$ Annexin V-FITC and $10 \mu \mathrm{l}$ PI were added and incubated in the dark for $15 \mathrm{~min}$. The cell apoptotic rate was analyzed using a flow cytometer (Becton Dickinson, USA).

\section{Statistical analysis}

Data are presented as mean \pm SEM for continuous variables. Comparison between groups were conducted using the one-way ANOVA or paired t-test. A value of $P<0.05$ (two-tailed) was considered as statistically significant. All analyses and draws were performed with Graphpad Prism 5.

\section{Results}

IL-1 $\beta$ increased gene and Protein expression of TRPA1 in rat chondrocytes

In the present study, the IL- $1 \beta$ was found to increase gene TRPA1 expression gradually in a dose-dependent manner: the expression of TRPA 1 increased up to the $10 \mathrm{ng} / \mathrm{ml}$ at $12 \mathrm{~h}$ or $24 \mathrm{~h}$ (Fig. 1a). In addition, to verify the translation

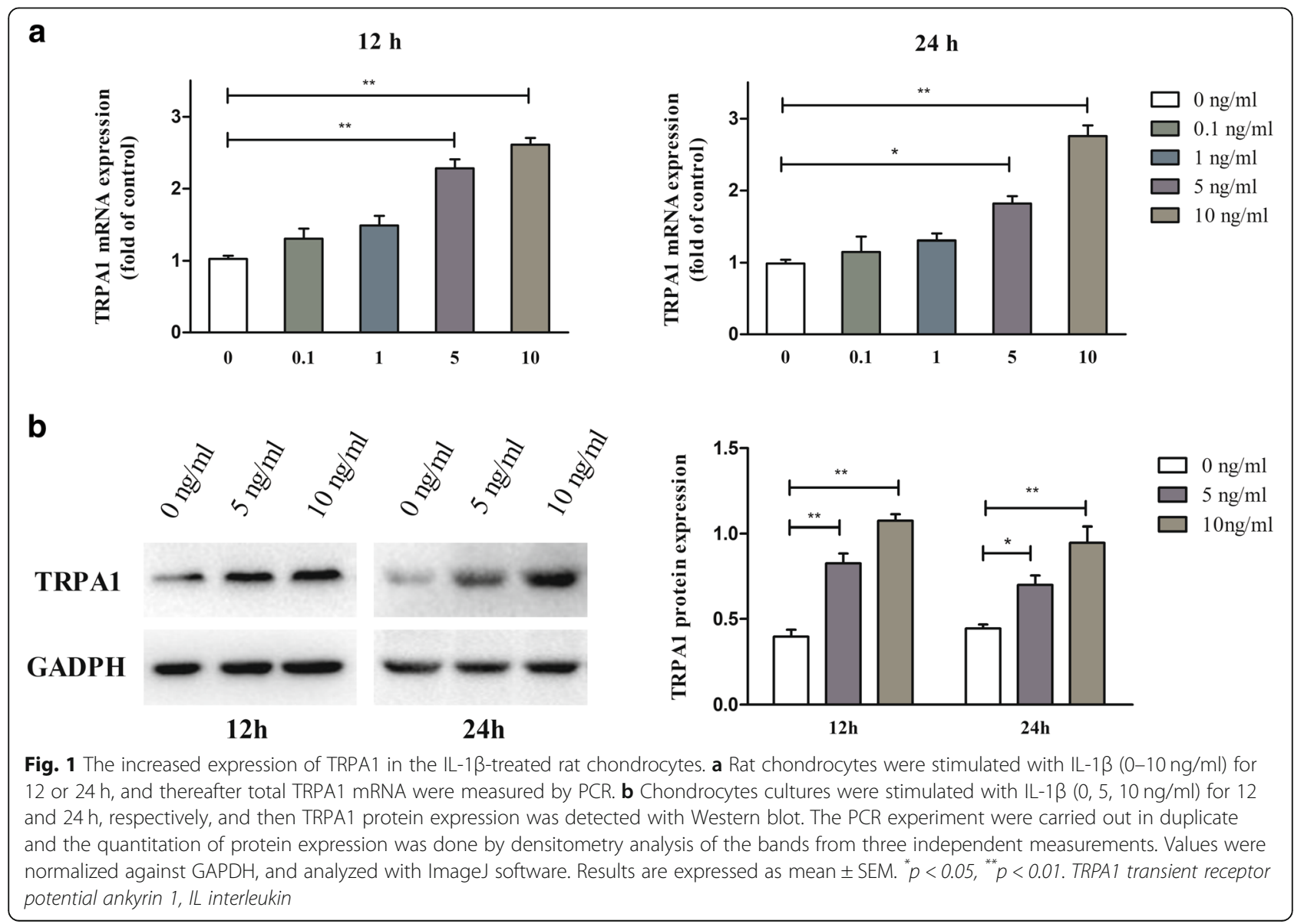


of TRPA1 mRNA into protein, total protein was also detected by western blot (Fig. 1b). As a result, expression of TRPA1 protein was also increased at the 5 and $10 \mathrm{ng} / \mathrm{ml}$ in IL-1 $\beta$-treated rat chondrocytes.

\section{IL-1 $\beta$ enhanced $\mathrm{Ca}^{2+}$ influx mediated by TRPA 1 in rat chondrocytes}

To confirm the calcium load mediated by TRPA1 channel in rat chondrocytes, $\mathrm{Ca}^{2+}$ influx measurement was introduced in the study. The results shown that $\mathrm{Ca}^{2+}$ influx in the IL-1 $\beta$-treated chondrocytes gradually enhanced with increasing concentrations of IL-1 $\beta(0,5,10$ $\mathrm{ng} / \mathrm{ml}$ ) for 12 or $24 \mathrm{~h}$ (Fig. 2), while the fluorescence intensity was significantly reduced after treatment with the specific TRPA1 antagonist, which indicated that TRPA1 may have a specific role in mediating extracellular $\mathrm{Ca}^{2+}$ influx in the IL-1 $\beta$-treated rat chondrocytes.

\section{IL-1 $\beta$-induced apoptosis was downregulated by}

\section{pharmacological inhibition of TRPA1}

Based on the result that IL-1 $\beta$ enhanced the $\mathrm{Ca}^{2+}$ influx mediated by TRPA1 channel, we aimed to further investigate the effect of TRPA1 inhibition on the apoptosis in the
IL-1 $\beta$-treated Chondrocytes. Remarkably, our results show that the percent of apoptotic cells significantly increases with increasing IL-1 $\beta$ concentrations, but is dramatically attenuated by pharmacological inhibition (Fig. 3).

\section{Effects of TRPA1 inhibition on the mitochondrial} membrane potential in the IL-1 $\beta$-treated chondrocytes Furtherly, we investigated the link between TRPA1 and mitochondrial membrane potential using JC-1 staining. As shown in Fig. 4, the mitochondrial membrane potential was reduced significantly in the IL-1 $\beta$-treated cells. Treatment with TRPA1 inhibition restored the mitochondrial membrane potential (Fig. 4).

\section{Effects of TRPA1 inhibition on the expression of apoptosis-associated proteins in the IL-1 $\beta$-treated chondrocytes}

The expression of BAX, cytochrome c release and cleaved PARP and the activity of caspase- 3 and caspase- 9 were detected to investigate the mechanisms of TRPA1 on IL-1 $\beta$-induced chondrocyte apoptosis. The results showed that IL-1 $\beta$ significantly increased BAX, cytochrome $\mathrm{c}$ release, cleaved PARP protein expression (Fig. 5b), as well as

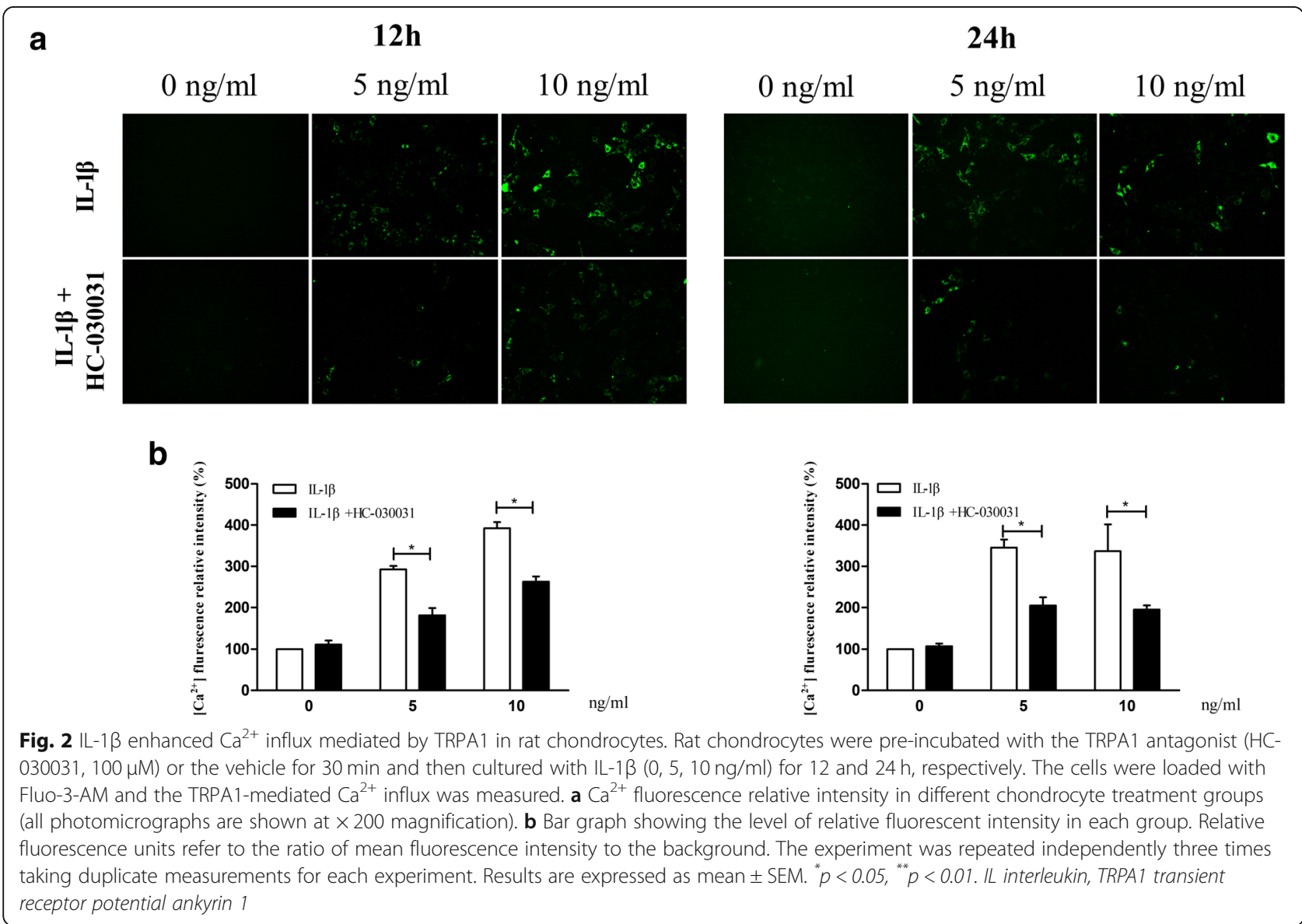




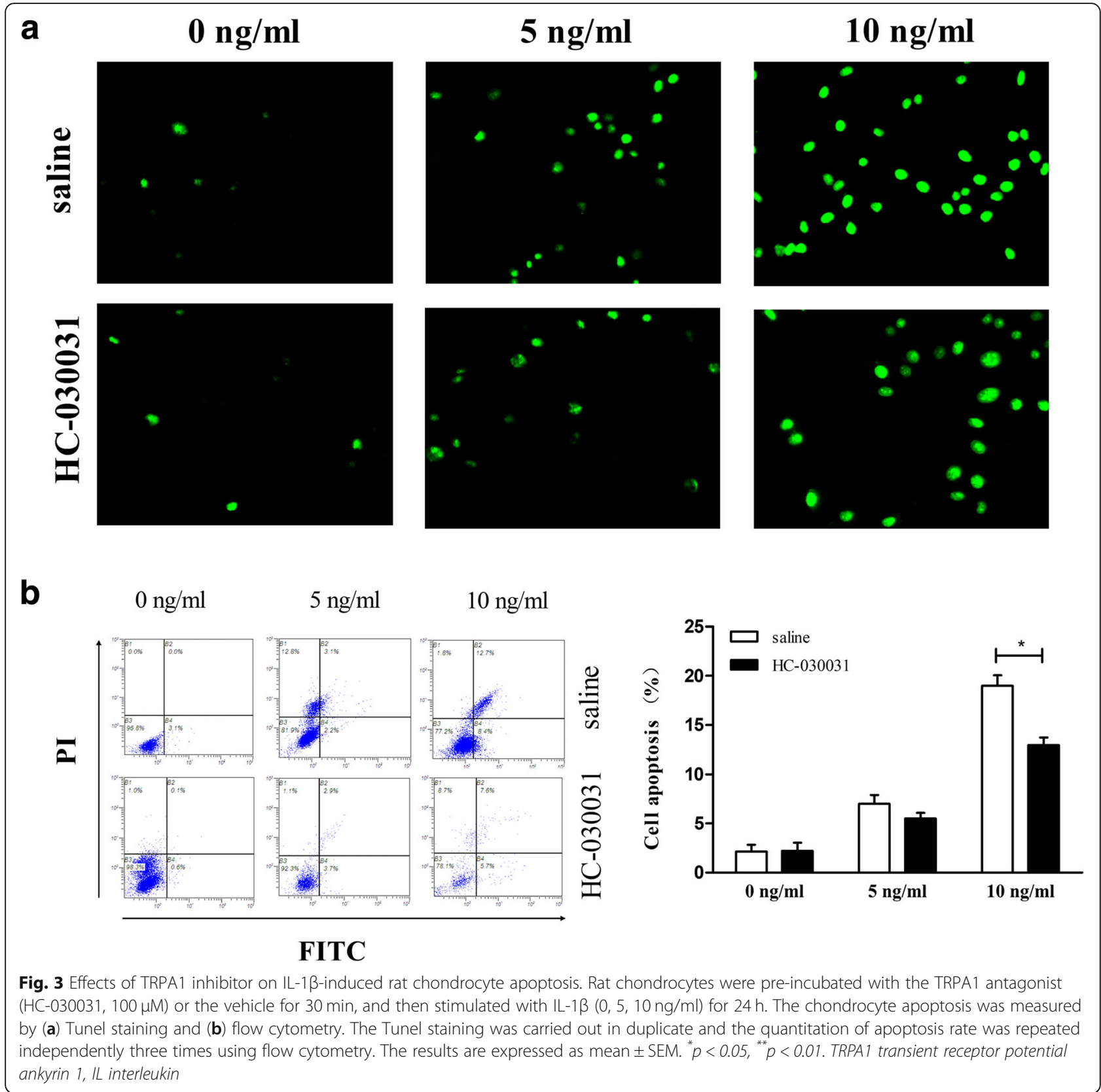

the caspase- 3 and 9 activity (Fig. 5a) in the chondrocytes. The treatment with TRPA1 inhibition evidently improved the abnormal expression of apoptosis-associated proteins (Fig. 5).

\section{Discussion}

Knee OA is a common degenerative joint disease, which pathologically characterized by the progressive degeneration of articular cartilage [2]. In OA joint, the activation of innate immune system markedly contributes to an increased expression of inflammatory cytokines, which play a crucial role in the initiation, maintenance, and perpetuation of osteoarthritic cartilage destruction [26, 27]. As a key inflammatory factor, IL- $1 \beta$ has been widely used as a chondrocyte apoptosis-inducing agent simulating the inflammatory environment of OA in vitro [28, 29]. In the current study, through establishing a model of IL-1 $\beta$-induced apoptosis, we found the IL- $1 \beta$ caused the increased functional expression of TRPA1 contributing to calcium overload, and inhibition of TRPA1 produced protective effect in IL-1 $\beta$-induced mitochondrial dysfunction and even apoptosis in rat chondrocytes.

TRPA1, firstly described in 1991, is a nonselective cation channel, especially permeable to $\mathrm{Ca}^{2+}$, widely expressed in 


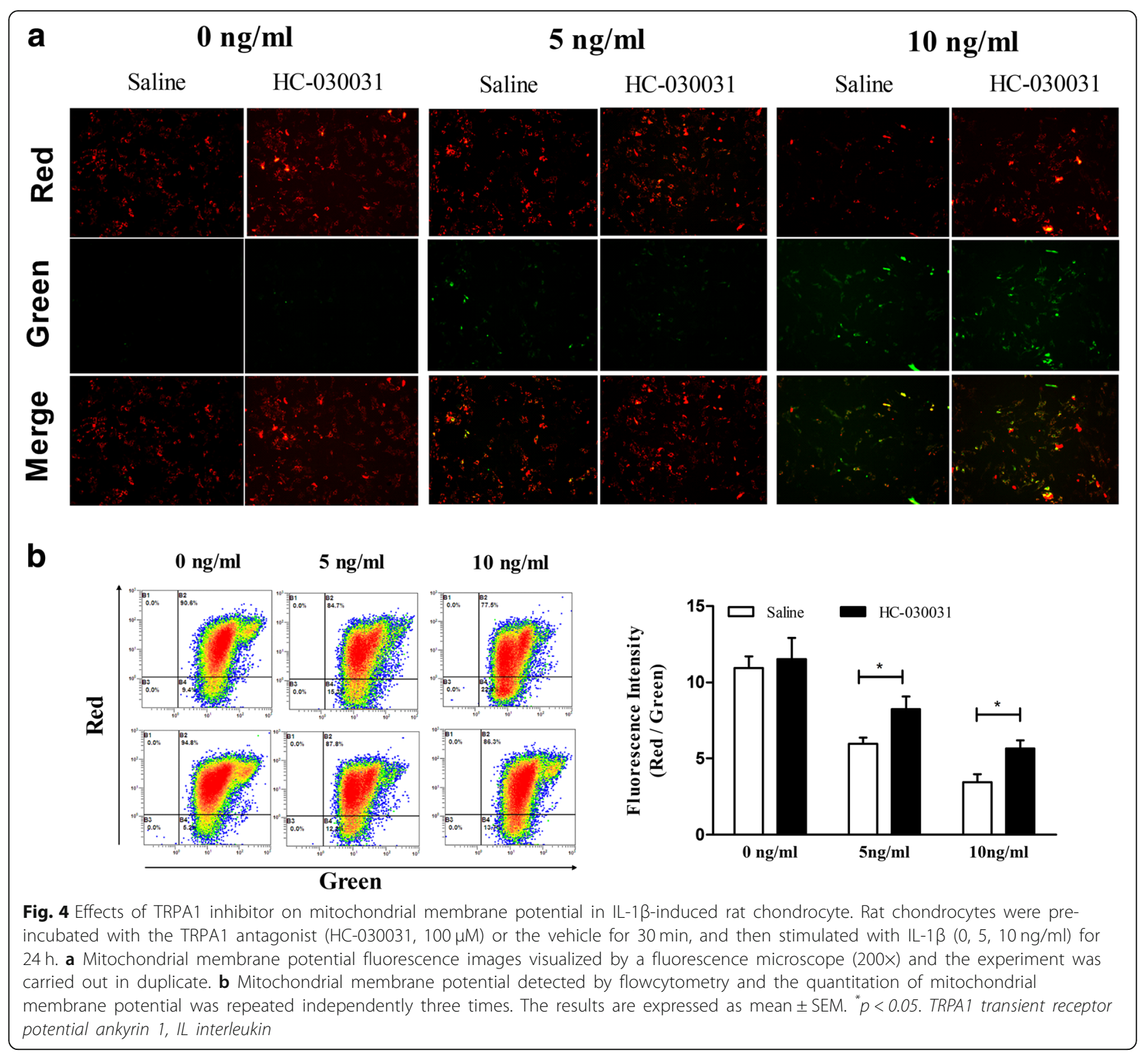

sensory neurons [30]. Recent years, emerging evidences suggested the expression of TRPA1 was also found in non-neuronal cells, synovial fibroblasts and even chondrocytes included [20, 24]. More recently, however, the inflammatory environment in OA joint, such as inflammatory cytokines, has been shown to upregulate the gene and protein expression of TRPA1. In our previous paper, we found that both LPS and its downstream factors TNF- $\alpha$, IL- $1 \beta$ increased the gene and protein expression of TRPA1 in human osteoarthritic synovial fibroblasts in a time-dependent and dose-dependent manner [20]. Besides, according to the recent paper by Moilanen et al. [24], the gene and protein expression of TRPA1 were significantly upregulated by inflammatory factors TNF- $\alpha$ and IL-1 $\beta$ in OA human chondrocytes. In the present study, we also found that IL-1 $\beta$ increased the gene and protein expression of TRPA1 in rat chondrocytes in a dose-dependent manner.

Interesting, not all of them identified the functionality of TRPA1 channel although the gene expression of TRPA1 have reported in some studies. In the present study, in order to identified the increased expression of TRPA1 is functional, we measured a $\mathrm{Ca}^{2+}$ influx using the TRPA1 antagonist in the IL-1 $\beta$-treated cells. As a result, we found the TRPA1 had a specific effect on mediating calcium influx in the IL-1 $\beta$-treated rat chondrocytes, which have fairly shown the expression and activation of TRPA 1 in rat chondrocytes. It is noteworthy that TRPA 1 can be activated by not only numerous exogenous pungent compounds but also agents formed endogenously in 


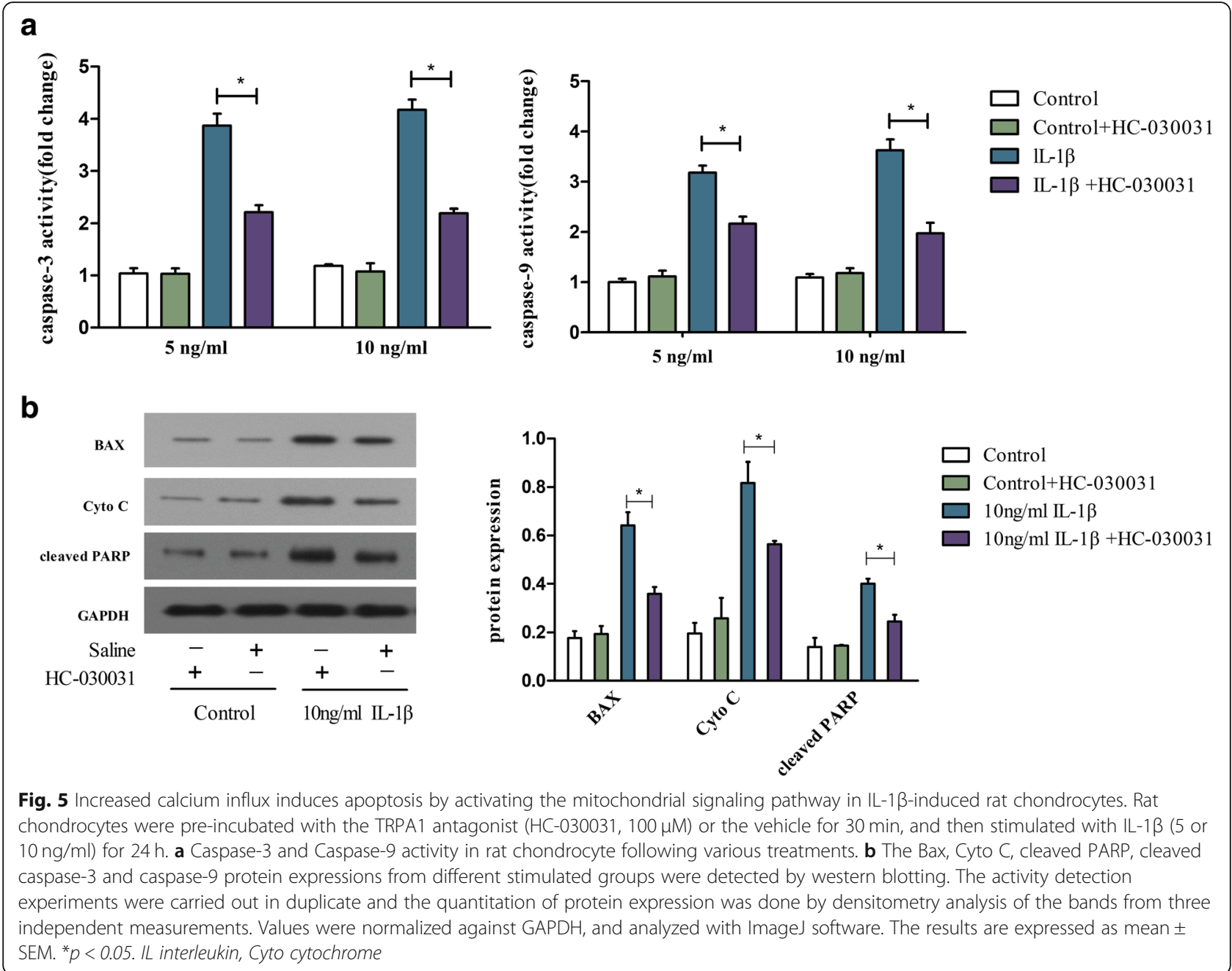

inflammatory reactions, such as nitric oxide [31], prostaglandins [32], and reactive oxygen species [33]. Moreover, in OA chondrocytes, the IL- $1 \beta$ has the ability to stimulate the production of mediators including nitric oxide [34], prostaglandin [35], and reactive oxygen species [36], which are involved in TRPA1 channel activation. Therefore, in these inflammatory cells, IL- $1 \beta$ maybe firstly upregulated the gene and protein expression of TRPA1, and then the increased expression of TRPA1 channel was activated by the endogenous agonists formed in inflammatory reactions, such as nitric oxide, prostaglandins, or reactive oxygen species. However, the detailed molecular mechanisms of the activation of TRPA1 channel demands further investigation remain.

It is well known that the role of $\mathrm{Ca}^{2+}$ influx through $\mathrm{Ca}^{2+}$-selective channels has been studied extensively, such as mediating DNA fragmentation and apoptosis [8-10]. In the present paper, we also testified that, in the IL-1 $\beta$-treated rat chondrocytes, calcium influx though the TRPA1channel could induce apoptosis, which agree with the previous studies indicating that TRPA1 inhibitor or deficiency could reduce the apoptosis of cardiomyocytes [37], oligodendrocytes [38], and even hippocampal neurons [39]. Apoptosis, a form of programmed cell death, is induced through two alternative pathways: extrinsic and intrinsic pathways, of which the intrinsic pathway is regulated by mitochondrial parameters [40]. Mitochondrial mediated apoptosis may initiate through the release of pro-apoptotic proteins into the cytosol due to mitochondrial dysfunction [41, 42]. Interesting, calcium influx was highlighted as being important in apoptosis attributing to its ability of inducing $\mathrm{Ca}^{2+}$ entry-dependent ROS production [8], mitochondrial depolarization and DNA fragmentation [9]. Our results suggested that calcium influx though the TRPA1 channel could induce mitochondrial depolarization in chondrocytes, and inhibition of TRPA1 repair the mitochondrial membrane potential. As a nonselective cation channel, TRPA1 channel also can permeate $\mathrm{Na}^{+}$and $\mathrm{K}^{+}$apart from $\mathrm{Ca}^{2+}$, and the $\mathrm{Na}^{+}$and $\mathrm{K}^{+}$permeation might also make 


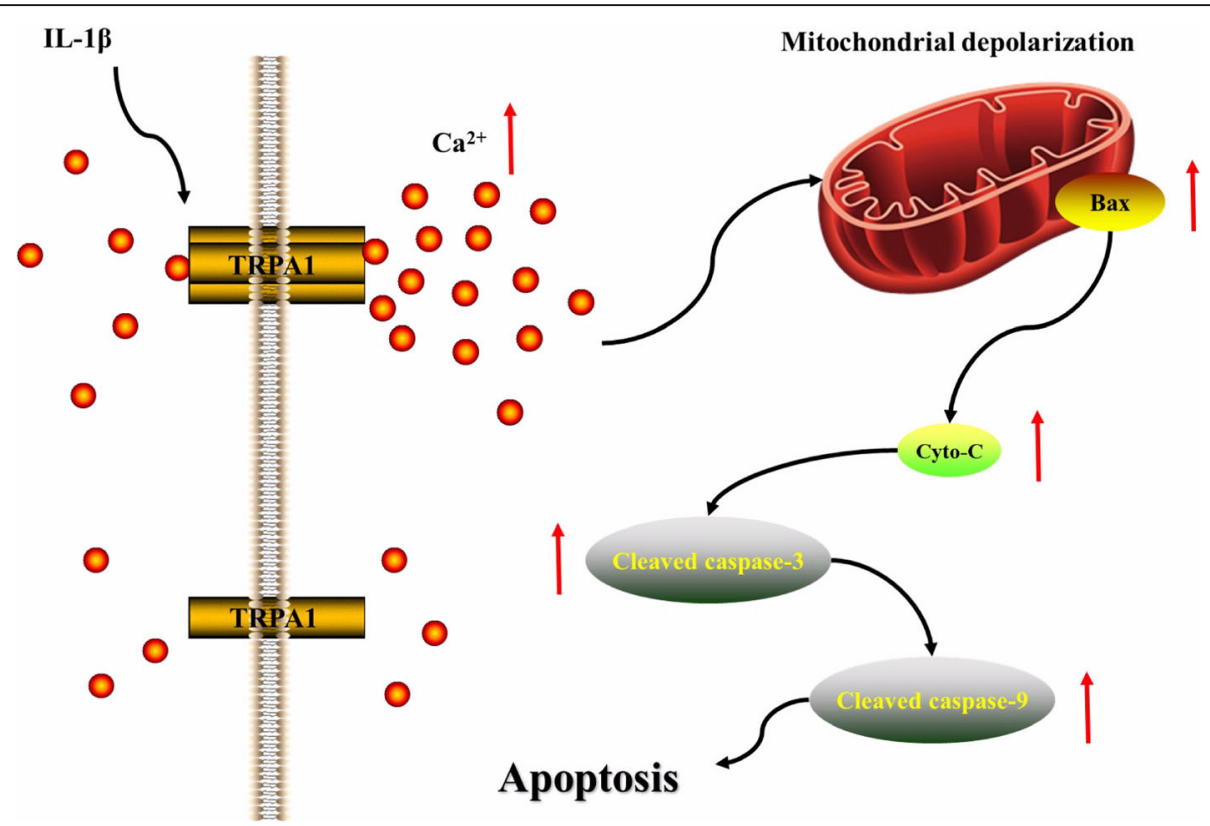

Fig. 6 Diagram of the signaling cascade involved in the effect of TRPA1-mediated $\mathrm{Ca}^{2+}$ influx on apoptosis in IL-1 $\beta$-induced rat chondrocytes. IL-1 $\beta$ caused increased functional expression of TRPA1, which contributed to massive $\mathrm{Ca}^{2+}$ influx. Due to cytosolic calcium overload, the mitochondrial membrane potential is significantly reduced, leading to the activation of the intrinsic pathway of apoptosis in rat chondrocytes. TRPA1 transient receptor potential ankyrin 1, IL interleukin, Cyto cytochrome

cells dysfunctional $[43,44]$. However, to date, the role of TRPA1-mediated $\mathrm{Na} 2+$ and $\mathrm{K}+$ permeation in the cell apoptosis may require future research which is beyond the scope of the current study. In addition, we also found that TRPA1 inhibitor could reduce the abnormal expressions of mitochondrial pro-apoptotic proteins including BAX, cytochrome c, PARP, caspase-3 and caspase-9. The present results suggest a potential possibility that TRPA1 channel involve in the process of mitochondrial dysfunction and intrinsic pathway in IL-1 $\beta$-induced apoptosis model.

In conclusion, we found the IL- $1 \beta$ caused increased functional expression of TRPA1, which contributed to massive $\mathrm{Ca}^{2+}$ influx. Due to cytosolic calcium overload, the mitochondrial membrane potential is significantly reduced, leading to the activation of the intrinsic pathway of apoptosis in rat chondrocytes (Fig. 6). These findings together with previous studies suggested the TRPA1 could be an effective target for the treatment of OA.

\section{Acknowledgements}

The authors wish to express their gratitude to all staffs in the medical research center of first college of clinical medicine, the Nanjing University of Chinese Medicine, Nanjing, China.

\section{Funding}

This research was supported by the National Natural Science Foundation of China $(81573993,81774334)$, Natural Science Foundation of Jiangsu province (SBK2017022501), Postgraduate Research \& Practice Innovation Program of Jiangsu Province (KYCX18_1551).

\section{Availability of data and materials}

All results and data are kept in the section for Departments of orthopedics, the Affiliated Hospital of Nanjing University of Chinese Medicine, Nanjing, China. These will be made available from the corresponding author on reasonable request.

\section{Authors' contributions}

SJY and JM conceived the study and drafted the manuscript. LZ and LD performed the isolation and culture of chondrocyte; SJY, XCL, ZQH and BX performed the rest cell experiments. All authors read and approved the final manuscript. All authors approved the final version and are accountable for all aspects of the manuscript.

\section{Ethics approval}

All animal protocols were approved (reference number: ACU170701) by the Animal Care and Use Committee of the Nanjing University of Chinese Medicine. All experiments were conducted in accordance with the National Institutes of Health Guidelines for the Care and Use of Laboratory Animals.

\section{Consent for publication}

Data was de-identified, after collection, and therefore there was no consent required from individual patients, according to the ethical approval.

\section{Competing interests}

The author declare that they have no competing interests

\section{Publisher's Note}

Springer Nature remains neutral with regard to jurisdictional claims in published maps and institutional affiliations.

Received: 27 August 2018 Accepted: 22 November 2018 Published online: 17 December 2018

\section{References}

1. Vina ER, Kwoh. Epidemiology of osteoarthritis: literature update. Curr Opin Rheumatol. 2018;30:160-7. 
2. Chen D, Shen J, Zhao W, Wang T, Han L, Hamilton JL, Im HJ. Osteoarthritis: toward a comprehensive understanding of pathological mechanism. Bone Res. 2017;17:16044.

3. Liu C, Wang B, Xiao L, Li Y, Xu L, Zhao Z, Zhang L. Protective effects of the pericellular matrix of chondrocyte on articular cartilage against the development of osteoarthritis. Histol Histopathol. 2018;33:757-64.

4. Sanchez C, Bay-Jensen AC, Pap T, Dvir-Ginzberg M, Quasnichka H, BarrettJolley R, Mobasheri A, Henrotin Y. Chondrocyte secretome: a source of novel insights and exploratory biomarkers of osteoarthritis. Osteoarthr Cartil. 2017;25:1199-209.

5. Hwang HS, Kim HA. Chondrocyte apoptosis in the pathogenesis of osteoarthritis. Int J Mol Sci. 2015;16:26035-54.

6. Pountos I, Giannoudis PV. Modulation of cartilage's response to injury: can chondrocyte apoptosis be reversed? Injury. 2017;48:2657-69.

7. Bouron A, Chauvet S, Dryer S, Rosado JA. Second messenger-operated calcium entry through TRPC6. Adv Exp Med Biol. 2016;898:201-49.

8. Kovac S, Domijan AM, Walker MC, Abramov AY. Seizure activity results in calcium- and mitochondria independent ROS production via NADPH and xanthine oxidase activation. Cell Death Dis. 2014;5:e1442.

9. Llorente-Folch I, Rueda CB, Pardo B, Szabadkai G, Duchen MR, Satrustegui J. The regulation of neuronal mitochondrial metabolism by calcium. J Physiol. 2015;593:3447-62.

10. Kim JY, Yu SJ, Oh HJ, Lee JY, Kim Y, Sohn J. Panaxydol induces apoptosis through an increased intracellular calcium level, activation of JNK and p38 MAPK and NADPH oxidase- dependent generation of reactive oxygen species. Apoptosis. 2011;16:347-58.

11. Hasan R, Zhang X. Ca ${ }^{2+}$ regulation of TRP ion channels. Int J Mol Sci. 2018; 19:E1256.

12. Mulier M, Vriens J, Voet T. TRP channel pores and local calcium signals. Cell Calcium. 2017;66:19-24.

13. Fliniaux I, Germain E, Farfariello V, Prevarskaya N. TRPs and $\mathrm{Ca}^{2+}$ in cell death and survival. Cell Calcium. 2018;69:4-18.

14. Sun Z, Han J, Zhao W, Zhang Y, Wang S, Ye L, Liu T. TRPV1 activation exacerbates hypoxia /reoxygenation-induced apoptosis in $\mathrm{H} 9 \mathrm{C} 2$ cells via calcium overload and mitochondrial dysfunction. Int J Mol Sci. 2014;15: 18362-80.

15. Hu F, Sun WW, Zhao XT, Cui ZJ, Yang WX. TRPV1 mediates cell death in rat synovial fibroblasts through calcium entry-dependent ROS production and mitochondrial depolarization. Biochem Biophys Res Commun. 2008;369: 989-93.

16. Wei Y, Zheng D, Guo X, Zhao M, Gao L, Bai L. Transient receptor potential channel, Vanilloid 5, induces chondrocyte apoptosis in a rat osteoarthritis model through the mediation of $\mathrm{Ca}^{2+}$ influx. Cell Physiol Biochem. 2018;46: 687-98.

17. Nilius B, Appendino G, Owsianik G. The transient receptor potential channel TRPA1: from gene to pathophysiology. Pflugers Arch. 2012;464:425-58.

18. Viana F. TRPA1 channels: molecular sentinels of cellular stress and tissue damage. J Physiol. 2016:594:4151-69.

19. Andrei SR, Ghosh M, Sinharoy P, Dey S, Bratz IN, Damron DS. TRPA1 ion channel stimulation enhances cardiomyocyte contractile function via a CaMKII-dependent pathway. Channels (Austin). 2017;11:587-603.

20. Yin S, Wang P, Xing R, Zhao L, Li X, Zhang L, Xiao Y. Transient receptor potential Ankyrin 1 (TRPA1) mediates lipopolysaccharide (LPS)-induced inflammatory responses in primary human osteoarthritic fibroblast-like Synoviocytes. Inflammation. 2018:41:700-9.

21. Bölcskei K, Kriszta G, Sághy É, Payrits M, Sipos É, Vranesics A, Berente Z, Ábrahám H, Komoly S and Pintér E. Behavioural alterations and morphological changes are attenuated by the lack of TRPA1 receptors in the cuprizone-induced demyelination model in mice. J Neuroimmunol. 2018;320:1-10

22. Horváth Á, Tékus V, Boros M, Pozsgai G, Botz B, Borbély É, Szolcsányi J, Pintér $E$, Helyes Z . Transient receptor potential ankyrin 1 (TRPA1) receptor is involved in chronic arthritis: in vivo study using TRPA1-deficient mice. Arthritis Res Ther. 2016;18:6.

23. Moilanen $\sqcup$, Hämäläinen $M$, Nummenmaa $E$, Ilmarinen $P$, Vuolteenaho $K$, Nieminen RM, Lehtimäki L, Moilanen E. Monosodium iodoacetate-induced inflammation and joint pain are reduced in TRPA1 deficient mice--potential role of TRPA1 in osteoarthritis. Osteoarthr Cartil. 2015:23:2017-26.

24. Nummenmaa E, Hämäläinen M, Moilanen LJ, Paukkeri EL, Nieminen RM, Moilanen T, Vuolteenaho K, Moilanen E. Transient receptor potential ankyrin
1 (TRPA1) is functionally expressed in primary human osteoarthritic chondrocytes. Arthritis Res Ther. 2016:18:185

25. Pan T, Shi X, Chen H, Chen R, Wu D, Lin Z, Zhang J, Pan J. Geniposide suppresses interleukin-1 $\beta$-induced inflammation and apoptosis in rat chondrocytes via the PI3K/Akt/NF-KB signaling pathway. Inflammation. 2018, 41:390-9.

26. Kapoor M, Martel-Pelletier J, Lajeunesse D, Pelletier JP, Fahmi H. Role of proinflammatory cytokines in the pathophysiology of osteoarthritis. Nat Rev Rheumatol. 2011:7:33-42

27. Takakubo Y, Barreto G, Konttinen YT, Oki H and Takagi M. Role of innate immune sensors, TLRs, and NALP3 in rheumatoid arthritis and osteoarthritis. $J$ Long Term Eff Med Implants. 2014;24:243-51.

28. Zheng X, Xia C, Chen Z, Huang J, Gao F, Li G, Zhang B. Requirement of the phosphatidylinositol 3-kinase/akt signaling pathway for the effect of nicotine on interleukin-1beta-induced chondrocyte apoptosis in a rat mode of osteoarthritis. Biochem Biophys Res Commun. 2012;423:606-12.

29. Zhao H, Zhang T, Xia C, Shi L, Wang S, Zheng X, Hu T, Zhang B. Berberine ameliorates cartilage degeneration in interleukin-1beta-stimulated rat chondrocytes and in a rat model of osteoarthritis via akt signalling. J Cell Mol Med. 2014;18:283-92.

30. Jaquemar D, Schenker T, Trueb B. An ankyrin-like protein with transmembrane domains is specifically lost after oncogenic transformation of human fibroblasts. J Biol Chem. 1999;274:7325-33.

31. Yoshida T, Inoue R, Morii T, Takahashi N, Yamamoto S, Hara Y, Tominaga M, Shimizu S, Sato Y, Mori Y. Nitric oxide activates TRP channels by cysteine Snitrosylation. Nat Chem Biol. 2006:2:596-607.

32. Zygmunt PM, Högestätt ED. TRPA1. Handb Exp Pharmacol. 2014;222:583-630.

33. Sullivan MN, Gonzales AL, Pires PW, Bruhl A, Leo MD, Li W, Oulidi A, Boop FA, Feng Y, Jaggar JH, Welsh DG and Earley S. Localized TRPA1 channel Ca2 + signals stimulated by reactive oxygen species promote cerebral artery dilation. Sci Signal. 2015;8:ra2.

34. Wang $Y$, Chen $Y$, Chen $Y$, Zhou B, Shan X, Yang G. Eriodictyol inhibits IL-1 $\beta$ induced inflammatory response in human osteoarthritis chondrocytes. Biomed Pharmacother. 2018;107:1128-34.

35. Fu Z, Chen Z, Xie Q, Lei H, Xiang S. Hesperidin protects against IL-1ßinduced inflammation in human osteoarthritis chondrocytes. Exp Ther Med. 2018:16:3721-7.

36. Liu M, Zhong S, Kong R, Shao H, Wang C, Piao H, Lv W, Chu X, Zhao Y. Paeonol alleviates interleukin-1 $\beta$-induced inflammatory responses in chondrocytes during osteoarthritis. Biomed Pharmacother. 2017;95:914-21.

37. Wang Z, Wang M, Liu J, Ye J, Jiang H, Xu Y, Ye D, Wan J. Inhibition of TRPA attenuates doxorubicin-induced acute cardiotoxicity by suppressing oxidative stress, the inflammatory response, and endoplasmic reticulum stress. Oxidative Med Cell Longev. 2018:28:5179468.

38. Sághy É, Sipos É, Ács P, Bölcskei K, Pohóczky K, Kemény Á, Sándor Z, Szőke É, Sétáló G Jr, Komoly S, Pintér E. TRPA1 deficiency is protective in cuprizone-induced demyelination a new target against oligodendrocyte apoptosis. Glia. 2016:64:2166-80.

39. Yazğan Y, Nazıroğlu M. Ovariectomy-induced mitochondrial oxidative stress, apoptosis, and calcium ion influx through TRPA1, TRPM2, and TRPV1 are prevented by $17 \beta$-estradiol, tamoxifen, and Raloxifene in the Hippocampus and dorsal root ganglion of rats. Mol Neurobiol. 2017;54:7620-38.

40. Musumeci G, Castrogiovanni P, Trovato FM, Weinberg AM, Al-Wasiyah MK, Alqahtani MH, Mobasheri A. Biomarkers of chondrocyte apoptosis and autophagy in osteoarthritis. Int J Mol Sci. 2015;16:20560-75.

41. Yu R, Zhang Y, Xu Z, Wang J, Chen B, Jin H. Potential antitumor effects of panaxatriol against DU-15 human prostate cancer cells is mediated via mitochondrial mediated apoptosis, inhibition of cell migration and sub-G1 cell cycle arrest. J BUON. 2018:23:200-4.

42. Wang K, Fan H, Chen Q, Ma G, Zhu M, Zhang X, Zhang Y, Yu J. Curcumin inhibits aerobic glycolysis and induces mitochondrial-mediated apoptosis through hexokinase II in human colorectal cancer cells in vitro. Anti-Cancer Drugs. 2015;26:15-24.

43. Rosendahl J, Braun HS, Schrapers KT, Martens H, Stumpff F. Evidence for the functional involvement of member of the TRP channel family in the uptake of $\mathrm{Na}+$ and $\mathrm{NH} 4+$ by the ruminal epithelium. Pflugers Arch. 2016:468:1333-52.

44. Earley S, Gonzales AL and Crnich R. Endothelium-dependent cerebral artery dilation mediated by TRPA1 and Ca2+-Activated K+ channels. Circ Res. 2009;104:987-94. 